\title{
Cost Effective Cholecystectemy in A Rural Tertiary Care Hospital
}

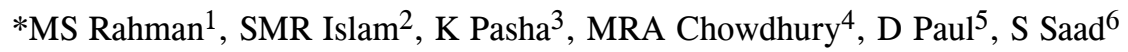 \\ $1 *$ Dr. Md. Shahinur Rahman, Assistant Professor, Department of surgery, Jahurul Islam Medical College Hospital \\ ${ }^{2}$ Dr. S M Rezaul Islam, Associate Professor, Department of surgery, Jahurul Islam Medical College Hospital \\ ${ }^{3}$ Dr. Kamal Pasha, Assistant Professor, Department of surgery, Jahurul Islam Medical College Hospital. \\ ${ }^{4}$ Dr. Md. Rashed Alam Chowdhury, Assistant Professor, Department of medicine, Jahurul Islam Medical College Hospital \\ ${ }^{5}$ Dr. Debabrata Paul, Assistant Registrar, Department of surgery, Jahurul Islam Medical College Hospital \\ ${ }^{6}$ Dr. Sanjeeda Saad, Post graduate resident, Department of Surgery, BSMMU \\ *Corresponding Author
}

Date of submissio: 6 June 2015 Date of acceptance: 15 Dec. 2015

\begin{abstract}
Background: Laparoscopic cholecystectomy is an accepted alternative to open cholecystectomy. This study was aimed to compare the results of laparoscopic and open cholecystectomy with respect to duration of operation, complications, hospital stay and cost.

Materials and Methods: This was a prospective type of study. 200 patients of gallstone disease were randomly selected. 100 patients underwent laparoscopic cholecystectomy and 100 underwent open cholecystectomy from July 2014 to June 2015 in surgery units of Jahurul Islam Medical College Hospital. Data regarding age, sex, operation time, complications, hospital stay and total cost were collected from patient and from hospital record. Then we compared the data of laparoscopic and open cholecystectomy.

Results: We found that mean operating time for laparoscopic cholecystectomy was 45 minutes and for open cholecystectomy was 60 minutes. There is no significant difference in incidence of complications between open and laparoscopic procedure. In our study, mean postoperative hospital stay for laparoscopic surgery was 3.5 days and in open cholecystectomy was 6 days. In this study total cost of laparoscopic surgery is less than open surgery though operation team charge is more in laparoscopic surgery.
\end{abstract}

Conclusion: Laparoscopic cholecystectomy is a safe and cost effective alternative to open cholecystectomy.

Key Words: Laparoscopic cholecystectomy, open cholecystectomy, cost effectiveness, gallstone disease

\section{Introduction}

Laparoscopic cholecystectemy is now the 'gold standard' for operative treatment of symptomatic gallstone disease ${ }^{1}$. First ever Laparoscopic cholecystectemy in Bangladesh was performed by Dr. Hashimoto and Dr. Sarder Nayeem on 20th December, 1991 in BIRDEM hospital ${ }^{2}$. Incidence of laparoscopic cholecystectomy is increasing. But still it is not satisfactory in district and upazila level hospitals. Main disadvantage of laproscopic cholecystectomy is increased incidence of bile duct injury. This can be avoided by proper training of the surgeons. Another barrier of gaining popularity of laparoscopic surgery is some doctors, health care personnel and patients still believe that laparoscopic

surgery is costly. It has been established that laparoscopic cholecystectomy could be performed at a treatment cost that is equal to or slightly less than that of open cholecystectomy, and with substantial cost savings to the patient and society due to the reduced loss of time from work ${ }^{3}$. The cost of laparoscopic cholecystectomy and open cholecystectomy has been compared in some randomized controlled trials with divergent results ${ }^{4}$.

The aim of this study was to analyze and compare the perioperative results of patients undergoing laparoscopic and open cholecystectomy concerning to duration of operation, complications, hospital stay and cost.

AKMMC J 2016; 7(1) : 20-22 


\section{Materials and Methods}

All patients having cholecystectomy at the Jahurul Islam Medical College Hospital, Bajitpur, Kishoreganj, Bangladesh, from July 2014 to June 2015 were prospectively registered in the study. Patients were eligible to participate in the trial comparing conventional open cholecystectomy and laparoscopic cholecystectomy according to exclusion and inclusion criteria. Approval of the Hospital Ethics Committee was obtained for the study. The patients signed the informed consent.

This is a private hospital. Its all services are payable. Here patients pay all the bills during discharge. The cost of hospital care included admission fee, seat rent, operation charge, medication cost, food, investigation charge, dressing charge and others. All patients were admitted in general paying beds. Operation team charge for laparoscopic cholecystectomy is Btk 9920.00 and for open cholecystectomy is Btk 8110.00 in this hospital.

We analyzed 200 patients, aged 16-70 years, diagnosed with gallstone disease selected for laparoscopic or open cholecystectomy. Out of them 100 patients underwent laparoscopic cholecystectomy and 100 patients underwent open cholecystectomy. Patients with choledocholithiasis, gall bladder perforation, empyema gall bladder, malignancy, seriously co-morbid patients requiring ICU or HDU care, those converted to open surgery and patients underwent concomitant surgeries were excluded from the study. Data such as age, sex, pre operative diagnosis, duration of surgery, concomitant diseases and use of prophylactic antibiotics were recorded. Per operative and early post operative complications were also recorded. Patient's total bill (operation charge, medication cost and others) were collected from the computerised data recording system of hospital at discharge. Length of postoperative hospital stay and the clinical conditions at the time of discharge also recorded.

\section{Results}

In this series, 100 patients underwent laparoscopic cholecystectemy and 100 patients underwent open cholecystectomy.65\% patients of laparoscopic surgery were in the age group 20 - 39 years, whereas $62 \%$ patients of open cholecystectomy were in the age group 40 - 59 yrs.
Table-I: Age distribution of patients ( years )

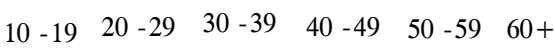

L aparoscopic

$\begin{array}{llllllll}\text { cholecystectemy } & 5 & 27 & 38 & 12 & 10 & 8\end{array}$

$\begin{array}{lllllll}\text { O pen cholecystectomy } & 3 & 10 & 15 & 30 & 32 & 10\end{array}$

In both group female patients are predominant. Sex distribution of patients are shown in Table 2.

Table-II: Sex distribution of patients

Male Female Male female ratio

$\begin{array}{lccc}\text { Laparoscopic cholecystectemy } & 15 & 85 & 1: 5.6 \\ \text { Open cholecystectomy } & 38 & 62 & 1: 1.6\end{array}$

Operating time in laparoscopic surgery was 30 to 60 minutes in 66 patients $(66 \%)$. Open surgery lasting 30 to 60 minutes were performed in 40 patients $(40 \%)$, significantly less when compared with laparoscopic surgery. We performed 34(34\%) laparoscopic cholecystectomy in the time interval 61-120 minutes, significantly less than $60(60 \%)$ open cholecystectomy in the same time interval (61180 minutes). Mean operating time for laparoscopic cholecystectomy was 45 minutes and for open cholecystectomy was 60 minutes.

Operative complications resulting from laparoscopic procedure occurred in 6 patients, 3 patients with port site infection. The remaining 3 patients who had complications were affected by duodenal injury, pneumonia, pulmonary oedema. In patients underwent open cholecystectomy complications occurred in 7 cases. Surgical site infection was the most frequent complication, diagnosed in 6 patients. One patient in each series developed cystic duct stump leakage presented as passage of bile through drain tube. Both were treated conservatively.

In patients underwent laparoscopic surgery maximum post operative hospial stay was 6 days and minimum was 2 days (average 3.5 days). In open surgery maximum post operative hospial stay was 14 days and minimum was 3 days (average 6 days). Operation team charge is more in laparoscopic surgery, but total cost is more in open surgery. 
Table-III: Operation costs in Btk. (Average per patient)

\begin{tabular}{lccc}
\hline Cholecystectomy & Operation charge & Medicine cost & Total cost \\
\hline Laparoscopic & 9920.00 & 8691.00 & 22738.00 \\
Open & 8110.00 & 15091.00 & 30685.00 \\
\hline
\end{tabular}

\section{Discussion}

The benefits of laparoscopic cholecystectomy has been established by many studies.In a study, it has been shown that the mean postoperative stay for an open cholecystectomy was 8 days compared with the 3 days for laparoscopic cholecystectomy5. In our study, mean postoperative hospital stay for laparoscopic surgery was 3.5 days and in open cholecystectomy was 6 days.

In our study mean operating time for open cholecystectomy was 15 minutes longer than laparoscopic cholecystectomy. In a similar study, the duration of open cholecystectomy was 22 min longer than laparoscopic cholecystectomy $(\mathrm{p}<0.001)^{6}$.

In this series there is no significant difference in complications between laparoscopic and open cholecystectomy. But study showed that frequency of complications was $13.5 \%$ for open cholecystectomy and $6.4 \%$ for laparoscopic cholecystectomy ${ }^{6}$. In this study total cost of laparoscopic surgery is less than open surgery though operation team charge is more in laparoscopic surgery. This is due to increase hospital stay and increase medication cost for open surgery. Literature showed that the average cost of laparoscopic cholecystectomy was lower than open cholecystectomy and laparoscopic cholecystectomy was more costeffective than open cholecystectomy (US\$ 995 vs. US\$ $1,048$, respectively $)^{6}$. In another study, the cost of the laparoscopic procedure was higher than that for open surgery ${ }^{7}$. This result does not support that of our study. But in another study we see that 5-year cumulative charges are lower for laparoscopic cholecystectomy than for open cholecystectomy (\$5,354 versus $\$ 5,525$ for 45 -year-old women; $\$ 6,036$ versus $\$ 6,830$ for 45 -year-old men), and the differences increase substantially with increasing age ${ }^{8}$.

There are some limitations of our study. Aged patients ( 40 to 59 years) with co morbidity and patients of acute cholecystitis are more in open cholecystectomy group. For these they had to pay extra cost for medication, investigation and monitoring. open cholecystectomy

\section{Conclusion}

Laparoscopic cholecystectomy is associated with a lower rate of postoperative complications, less operating time and shorter average hospital stay than open cholecystectomy. The total costs of laparoscopic cholecystectomy also less that of the open procedure. The difference in cost is attributable to the considerably shorter postoperative stay and less medication cost in laparoscopic procedure. So patients of gallstone disease of this rural area can be offered laparoscopic cholecystectomy as a cost effective procedure.

Conflict of interest: We have no conflict of interest.

\section{References}

1. Norman SW, Christopher JKB, P Ronan OC. Bailey and Love's Short Practice of Surgery. 25th edition.2008.

2. Mahbubul A. Laparoscopic surgery: History and present status in Bangladesh.BJE. Dec 2011.

3. Nilsson E, Ros A, Rahmqvist M, Backman K, Carlsson P. Cholecystectomy: costs and health-related quality of life: a comparison of two techniques. Int J Qual Health Care. 2004;16: 473-82.

4. Calvert NW, Troy GP, Johnson AG. Laparoscopic cholecystectomy: a good buy? A cost comparison with small-incision (mini) cholecystectomy.Eur J Surg. 2000; 166: 782-6.

5. Kesteloot K, Penninckx F. The costs and effects of open versus laparoscopic cholecystectomies. Health Economics 1993; 2: 303-312.

6. Fajardo R1, Valenzuela JI, Olaya SC, Quintero G, Carrasquilla G, Pinzón CE, et al. Cost-effectiveness of laparoscopic versus open cholecystectomy. Biomedica. 2011 Oct-Dec; 31(4): 514-24.

7. N. El-Fellah, A. Rogdakis,P. Giannakakis , V. Karambas G. Gratsias, P Bouras,et al. Cost of laparosopic and open cholecystectomy in a general hospital from the point of view of health care budgeting .June 2011, Volume 83, Issue 3, pp 139-147.

8. Eric B, Henry AP,Keith DL. Cost effectiveness of laparoscopic cholecystectomy versus open cholecystectomy. The American Journal of Surgery (Impact Factor: 2.29). 05/1993; 165(4): 466-71. 